\title{
TWISTING SPUN KNOTS
}

BY

E. C. ZEEMAN

1. Introduction. In [5] Mazur constructed a homotopy 4-sphere which looked like one of the strongest candidates for a counterexample to the 4-dimensional Poincaré Conjecture. In this paper we show that Mazur's example is in fact a true 4-sphere after all. This raises the odds in favour of the 4-dimensional Poincaré Conjecture.

The proof involves a smooth knot of $S^{2}$ in $S^{4}$ with unusual properties. Firstly, the group of the knot is

$$
\pi_{1}\left(S^{4}-S^{2}\right)=G \times Z,
$$

where $Z=$ integers, and $G=$ binary dodecahedral group. Since $G$ has order 120, this answers affirmatively a question of Fox [3, Problems 33 and 34] asking if the group of an $S^{2}$ knot in $S^{4}$ could have elements of even order.

Secondly, the complement $S^{4}-S^{2}$ of the knot is a fibre bundle over $S^{1}$ with fibre the punctured dodecahedral space, and group $Z_{5}$ (= cyclic of order 5). The dodecahedral space $M^{3}$ is defined to be the quotient space $M^{3}=S^{3} / G$, where $G$ is embedded as a subgroup of $S^{3}$ by the double covering

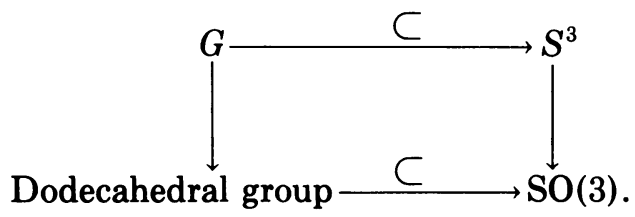

The dodecahedral space $M^{3}$ is the only known homology 3-sphere with nontrivial finite fundamental group $[9, \S 62]$. By punctured $M^{3}$, or more briefly punc $M^{3}$, we mean a space homeomorphic to $M^{3}$ minus a point; and by bounded punctured $M^{3}$ we mean a space homeomorphic to $M^{3}$ minus a (tame) open 3-cell.

The knotted $S^{2}$ in $S^{4}$ is obtained by spinning a trefoil knot in the manner of Artin [1], with the additional refinement that we twist it 5 times as it spins. The precise formulation of "twisting" and "spinning" is given in $\$ 6$, together with a more elaborate intuitive description in $\$ 4$.

More generally we give a recipe for $k$-twist-spinning any smooth $S^{n-2}$ knot in $S^{n}$. The result is a smooth $S^{n-1}$ knot in $S^{n+1}$, whose complement is a bundle over $S^{1}$, with group $Z_{k}$ and fibre punc $M^{n}$, where $M^{n}$ is the $k$-fold cyclic branched covering of $S^{n}$, branched over the original $S^{n-2}$ knot. More-

Received by the editors November 18, 1963. 


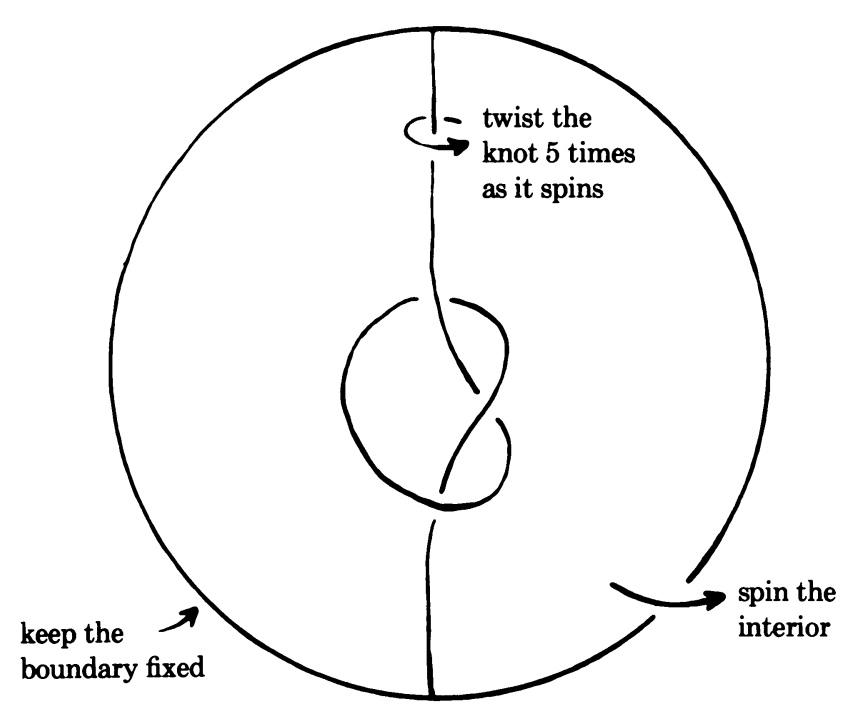

FIgURE 1

over $S^{1}$ acts on $S^{n+1}$ so as to leave the $S^{n-1}$ invariant (setwise not pointwise), and map the complement fibrewise. In particular if $k= \pm 1$ the result is unknotted.

A corollary to the theorem is that the punctured dodecahedral space can be differentially embedded in $S^{4}$. On the other hand Wall has shown that the (unpunctured) dodecahedral space $M^{3}$ cannot be tamely embedded in $S^{4}$. His proof uses the fact that $M^{3}$ is the boundary of an algebraic variety of index 8, with Stiefel-Whitney class $W_{2}=0$. If one could embed $M^{3}$ in $S^{4}$, then each component of the complement would be homologically trivial, and glueing one of them onto the variety would give a closed 4-manifold of index 8, contradicting Rohlin's theorem [7], which says the index must be divisible by 16 .

Another application is the differential embedding of the punctured lens spaces $L(p, q), p$ odd, in $S^{4}$, confirming a conjecture of Epstein [2]. This application uses a theorem of Schubert [8, Satz 6] which says that these lens spaces occur as double branched coverings of certain knots, and so we obtain the embeddings by 2-twist-spinning the knots. On the other hand an (unpunctured) lens space cannot be embedded in $S^{4}$, because the cohomology ring cannot be split into two components compatible with both the Bockstein coboundary operator and Alexander duality. In contrast Epstein [2] has shown that when $p$ is even the lens spaces $L(p, q)$ cannot be differentially embedded in $S^{4}$, whether punctured or unpunctured. His proof uses a Thom construction and Pontryagin classes. It is interesting that Schubert's technique [8] gives all the lens spaces occurring as branched 
coverings, but luckily we do not have a contradiction here, because when $p$ is even the branching is over a link rather than a knot.

The $k$-twist-spinning process does not by any means generate all knots, because for instance if the process is applied to $S^{0} \subset S^{2}$ then we obtain only the trivial knot of $S^{1}$ in $S^{3}$; but it does look like a promising construction for providing counterexamples.

2. Mazur's example [5]. Let $M^{3}$ be the dodecahedral space. Choose a homeomorphism $h$ of $M^{3}$ with the properties:

(a) $h$ is orientation preserving,

(b) $h$ has period 5 ,

(c) $h$ has a fixed point,

(d) $h_{*}$ kills the fundamental group (i.e., adding the relations $h_{*} x=x$, $x \in \pi_{1}\left(M^{3}\right)$ reduces $\pi_{1}\left(M^{3}\right)$ to zero).

Mazur gives an explicit example of $h$ which is equivalent to the following description. Represent $M^{3}$ as a dodecahedron with the opposite faces glued together $[9, \S 62]$. Then $h$ is the homeomorphism induced by rotating the dodecahedron through an angle $2 \pi / 5$ about a diameter perpendicular to a pair of faces. This particular $h$ has a whole circle of fixed points. We discuss other choices of $h$ in $\$ 8$.

Let $I$ denote the unit interval. Now glue the ends of $M^{3} \times I$ together by $h$ to form a bundle

$$
M^{3} \longrightarrow M^{4} \longrightarrow S^{1}
$$

with group $Z_{5}$. The fixed point gives a cross-section to the bundle, which we now surger out. More precisely choose a tubular neighbourhood $D^{3} \times S^{1}$ of this cross-section and replace it by $S^{2} \times D^{2}$, thereby defining a manifold

$$
Q^{4}=\left(M^{4}-D^{3} \times S^{1}\right) \cup S^{2} \times D^{2} \text {. }
$$

We shall show in $\$ \$ 4$ and 6 that one of these choices gives $Q^{4}=S^{4}$. The other choices are discussed in $\$ 8$.

Meanwhile we can check that $Q^{4}$ is a homotopy 4-sphere by showing it to be a homology 4-sphere and simply connected. The homology property follows from the Mayer-Vietoris sequence and the fact that $M^{4}$ is a homology torus $S^{3} \times S^{1}$. To show $Q^{4}$ simply connected, we first look at $\pi_{1}\left(M^{4}\right)$. From the homotopy exact sequence of the fibering we have $\pi_{1}\left(M^{4}\right)$ sitting in a short exact sequence,

$$
0 \longrightarrow G \longrightarrow \pi_{1}\left(M^{4}\right) \longrightarrow 0 .
$$

The action of a generator $z \in Z$ is the isomorphism $h_{*}$ on $G=\pi_{1}\left(M^{3}\right)$ induced by the homeomorphism $h$. By explicit computation we can choose generators $x, y$ of $G$, and lift $z$ back into $\pi_{1}\left(M^{4}\right)$ so that 


$$
\pi\left(M^{4}\right)=\left\{x, y, z ; x^{5}=(x y)^{3}=(x y z)^{2}, z^{-1} x z=y, z^{-1} y z=y x^{-1}\right\} .
$$

The effect of the surgery is to add the relation $z=1$, which kills the whole group. Therefore $\pi_{1}\left(Q^{4}\right)=0$, and so we have shown that $Q^{4}$ is a homotopy 4-sphere.

The knot involved in $Q^{4}$ is the core of the surgery

$$
S^{2}=S^{2} \times 0 \subset S^{2} \times D^{2} \subset Q^{4} \text {. }
$$

There are homeomorphisms

$$
\begin{aligned}
Q^{4}-S^{2} & \cong Q^{4}-S^{2} \times D^{2} \\
& =M^{4}-D^{3} \times S^{1} \\
& \cong M^{4}-S^{1}
\end{aligned}
$$

where $S^{1}$ denotes the cross-section through the fixed single point of $h$. Removing this cross-section leaves the bundle

$$
\text { punc } M^{3} \longrightarrow\left(Q^{4}-S^{2}\right) \longrightarrow S^{1} \text {. }
$$

This shows the fibering of the complement of the knot. To work out the fundamental group we observe that $\pi_{1}$ (punc $\left.M^{3}\right)=\pi_{1}\left(M^{3}\right)=G$, and so $\pi_{1}\left(Q^{4}-S^{2}\right)=\pi_{1}\left(M^{4}\right)$. Now the above exact sequence for $\pi_{1}\left(M^{4}\right)$ does not split, and so it is not clear that $\pi_{1}\left(M^{4}\right) \cong G \times Z$. To prove this we modify Mazur's proof slightly (we need to modify his proof, because in his paper he confuses $G$ with the dodecahedral group and therefore mistakenly assumes that $G$ is simple). Let $g=x y^{-1} x^{-1} \in G$. Then by computations we can show that the inner automorphism induced on $G$ by $g$ coincides with the action of $z$ on $G$ in $\pi_{1}\left(M^{4}\right)$. Therefore if $z_{*}=g^{-1} z$, then $z_{*}$ is a generator of $\pi_{1}\left(M^{4}\right)$ of infinite order that commutes with $G$. Therefore if $Z_{*}$ is the free cyclic subgroup generated by $z_{*}$ we have $\pi_{1}\left(M^{4}\right)=G \times Z_{*}$.

Finally we look at the action of $S^{1}$ on $Q^{4}$. To begin with, $S^{1}$ acts on the bundle $M^{4} \rightarrow S^{1}$ by rotating the base $S^{1}$ five times and acting on $M^{4}$ fibrewise. The cross-section is an invariant circle and is rotated on itself five times. Provided the tubular neighbourhood is chosen correctly, then the induced action on the boundary $S^{2} \times S^{1}$ of the tubular neighbourhood is the product of five rotations of $S^{1}$ with one rotation of $S^{2}$ about a diameter. This extends to a similar product action of $S^{1}$ on $S^{2} \times D^{2}$, and hence gives an action of $S^{1}$ on $Q^{4}$. The action of $S^{1}$ on the knot $S^{2}=S^{2} \times 0$ is rotation once about a diameter.

REMARK. In his paper Mazur makes the mistake of thinking that $S^{1}$ leaves the knot pointwise fixed as opposed to setwise fixed (the mistake occurs on page 248 , line 23 , in the words "it is also clear that"). This leads him to claim erroneously that the Smith conjecture [10] in higher dimensions is false, i.e., that there exists a periodic map of $S^{n}$ with fixed point 
set a knotted $S^{n-2}$. As far as I know $\left({ }^{1}\right)$ this conjecture is still open for all $n \geqq 3$.

3. A digression on the trefoil knot. I personally found it hard to visualise how the complement of a knot could be fibered so beautifully, until I heard a talk by John Stallings on Neuwirth knots (see [6] and [11]). A Neuwirth knot is an ordinary $S^{1}$ knot in $S^{3}$ whose group has finitely generated commutator subgroup.

Theorem (Neuwirth [6] and Stallings [11]). Let $S^{1} \subset S^{3}$ be a Neuwirth knot of genus g. Then:

1. The commutator subgroup of $\pi_{1}\left(S^{3}-S^{1}\right)$ is free on $2 g$ generators, and

2. if $M_{g}^{2}$ denotes the orientable surface of genus $g$, there is a fibre bundle

$$
\text { (punc } \left.M_{g}^{2}\right) \longrightarrow\left(S^{3}-S^{1}\right) \longrightarrow S^{1} \text {. }
$$

The closure of a fibre is a minimal surface spanning the knot.

For the benefit of readers who enjoy visualising such a fibering and who wish for a more explicit picture in 3-dimensions in order to gain insight into the 4-dimensional Mazur example and the $n$-dimensional theorem in $\$ 6$, let me now digress for a little while to describe in detail the fibering of the complement of the trefoil knot. In this case the fibre will be a punctured torus.

Represent $S^{3}$ as the join of two circles $S^{3}=S_{1}^{1} * S_{2}^{1}$. Let $S^{1}$ act on $S^{3}$ by rotating $S_{1}^{1}$ twice and $S_{2}^{1}$ thrice, and joining. More precisely if $\psi \in S^{1}$, $\theta_{i} \in S_{i}^{1}$, and $t \in I$ is the join parameter, then $\psi$ acts by mapping

$$
\left(\theta_{1}, t, \theta_{2}\right) \longrightarrow\left(\theta_{1}+2 \psi, t, \theta_{2}+3 \psi\right) \text {. }
$$

In terms of unit quaternions this is the action $z_{1}+j z_{2} \rightarrow e^{2 i \downarrow} z_{1}+j e^{3 i \downarrow} z_{2}$, where $z_{1}$ and $z_{2}$ are complex numbers. The trefoil knot lies on the halfway torus $t=1 / 2$, and is given by $3 \theta_{1}=2 \theta_{2}$. The action of $S^{1}$ rotates the trefoil on itself once.

We shall now describe a minimal surface $T^{2}$ spanning the knot, which will be a bounded punctured torus. The interior of $T^{2}$ is a punctured torus with the property that the action of $S^{1}$ on the interior of $T^{2}$ gives a family of punctured tori fibering $S^{3}$ minus the trefoil. If $\psi=\pi / 3$ in $S^{1}$, then the action of $\psi$ is to map each fibre onto itself by a homeomorphism of period 6 , and so the group of the bundle is $Z_{6}$. So now let us describe $T^{2}$.

$T^{2}$ will be the union of five disks $A_{1}, A_{2}, B_{1}, B_{2}, B_{3}$ as follows. $A_{1}, A_{2}$ will be the two disks in the solid torus $t \geqq 1 / 2$ given by $\theta_{2}=0$ and $\theta_{2}=\pi$. The action of $S^{1}$ on $A_{1}$ fibres the solid torus $t \geqq 1 / 2$ by the disks $\theta_{2}=$ constant. In particular if $\psi=\pi / 3$, then the action of $\psi$ is to interchange $A_{1}$ and $A_{2}$.

$\left({ }^{1}\right)$ Added in proof. C. H. Giffen has now shown that the Smith conjecture is false for all $n \geqq 4$, by using the branched coverings of twisted spun knots. 
If $\psi=2 \pi / 3$ the action of $\psi$ is to map each disk onto itself with a rotation of $4 \pi / 3$.

The description of the $B$ 's is a little harder. Let $R \times D^{2}$ be the solid cylinder that is the universal cover of the other solid torus $t \leqq 1 / 2$. Coordinates in $R \times D^{2}$ are given by $\tilde{\theta}_{1} \times\left(t, \theta_{2}\right)$ where $\tilde{\theta}_{1} \in R$ and $\theta_{1}=\tilde{\theta}_{1}$ reduced modulo $2 \pi$, while $0 \leqq t \leqq 1 / 2, \theta_{2} \in S_{2}^{1}$ and $\left(t, \theta_{2}\right)$ are polar coordinates in $D^{2}$. The trefoil lifts to the double helix on the surface of the cylinder given by

$$
t=1 / 2, \quad 3 \tilde{\theta}_{1}=2 \theta_{2} \text { modulo } 2 \pi .
$$

The boundaries of the disks $A_{1}, A_{2}$ lift into the straight lines

$$
t=1 / 2, \quad \theta_{2}=0, \pi \text {. }
$$

Given $\lambda, 0 \leqq \lambda \leqq \pi$, let $I_{\lambda}$ be the straight interval in $R \times D^{2}$ joining the points $2 \lambda / 3 \times(1 / 2, \lambda)$ and $2(\pi-\lambda) / 3 \times(1 / 2,2 \pi-\lambda)$.

Define $\tilde{B}_{1}$ to be the ruled surface (see Figure 2) $\tilde{B}_{1}=\bigcup_{0 \leqq \lambda \leqq \pi} I_{\lambda}$, and let $B_{1}$ be the image of $\tilde{B}_{1}$ under the covering projection. The boundary $\partial B_{1}$ consists of 4 arcs, one contained in each of $\partial A_{1}, \partial A_{2}$ and the other two contained in the trefoil. The action of $S^{1}$ on the solid torus $t \leqq 1 / 2$ lifts to the isometric action of $R$ on the solid cylinder given by screwing the solid cylinder along the double helix. The images of $\tilde{B}_{1}$ under this action consist of a family of disks which are disjoint except for where their boundaries intersect the double helix. (This can be shown by elementary Euclidean geometry.) Therefore the disks minus the double helix fibre the solid cylinder minus the double helix. Down below in the solid torus $t \leqq 1 / 2$; the images of $B_{1}$ under the action of $S^{1}$ induce a fibering of the solid torus minus the trefoil. In particular if $\psi=\pi / 3$, let $B_{2}=\psi B_{1}, B_{3}=\psi B_{2}$; then $B_{1}=\psi B_{3}$ and so $\psi$ permutes the three disks.

If $\psi=\pi$ then $\psi$ maps each disk $B_{i}$ onto itself with a rotation of $\pi$ about the centre. Since $\partial\left(B_{1} \cup B_{2} \cup B_{3}\right)=\partial\left(A_{1} \cup A_{2}\right) \cup$ the trefoil, we have that $T^{2}=A_{1} \cup A_{2} \cup B_{1} \cup B_{2} \cup B_{3}$ is a 2-manifold spanning the trefoil. Since $T^{2}$ is orientable with Euler characteristic -1 it must be a bounded punctured torus, and so the interior is a punctured torus. By construction the action of $S^{1}$ on the interior of $T^{2}$ generates a fibering of $S^{3}$ minus the trefoil. In particular if $\psi=\pi / 3$ then the action of $\psi$ maps each fibre to itself so as to interchange the $A$ disks and permute the $B$ disks, i.e., give a homeomorphism of period 6 . Hence the bundle

$$
\text { (punc torus) } \longrightarrow\left(S^{3}-\text { trefoil }\right) \longrightarrow S^{1}
$$

has group $Z_{6}$.

REMaRK 1. Not every Neuwirth knot has finite bundle group.

REMARK 2. Comparing $\$ \$ 2$ and 3 we conclude: Had we started with a torus $M^{2}$ and the homeomorphism $h: M^{2} \rightarrow M^{2}$ of period 6 keeping a point 
(i) Minimal surface spanning trefoil.

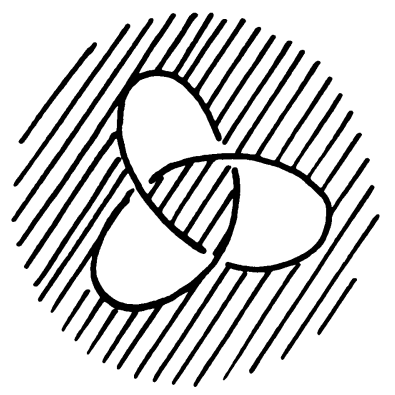

(iii) Solid torus $t \leqq \frac{1}{2}$. (ii) Minimal surface as union of five disks.

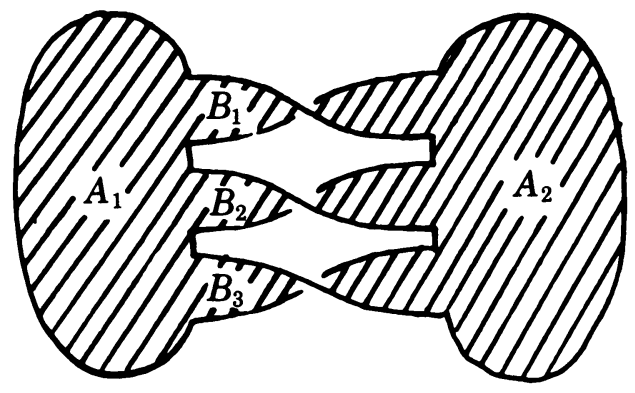

(iv) Universal cover of the solid torus $t \leqq \frac{1}{2}$.
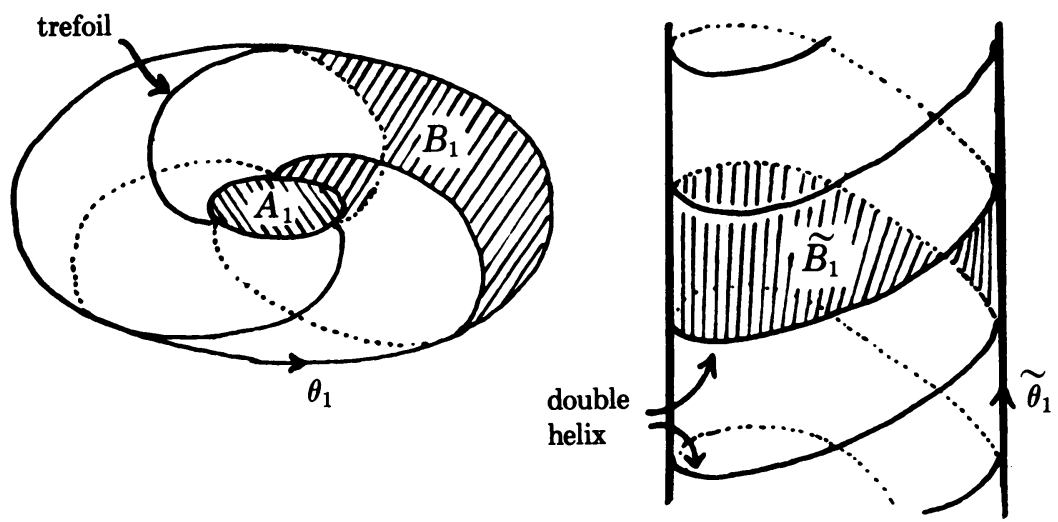

Figure 2

fixed, and then applied Mazur's construction (with suitable choice of tubular neighbourhood) then we should have recovered the sphere $S^{3}$, and the knot involved would be the trefoil. This gives the intuitive picture of Mazur's construction.

REMARK 3. It is interesting to note that although the trefoil and the action of $S^{1}$ on $S^{3}$ are algebraic, yet the fibering of the complement does not seem to be expressible algebraically (only differentially or piecewise algebraically). This is brought out more strongly in Lemma 2 below, where the existence of the fibering turns out to be a homotopy property.

Before leaving our digression we mention a 4-dimensional corollary to the Stallings' theorem. 
Corollary. Let $S^{2} \subset S^{4}$ be the suspension of a Neuwirth knot of genus $g$. Let $H_{2 g}^{3}$ be a handlebody of genus $2 g$, formed by glueing $2 \mathrm{~g}$ solid handles onto a 3-ball. Then there is a fibre bundle

$$
\text { (int } \left.H_{2 g}^{3}\right) \longrightarrow\left(S^{4}-S^{2}\right) \longrightarrow S^{1} .
$$

Proof. Since $S^{2} \subset S^{4}$ is the suspension of $S^{1} \subset S^{3}$ we have $S^{4}-S^{2}$ $\cong\left(S^{3}-S^{1}\right) \times R$. Since $S^{3}-S^{1}$ is fibered by punc $M_{g}^{2}$ it follows that $S^{4}-S^{2}$ is fibered by (punc $M_{g}^{2}$ ) $\times R$. To see that (punc $M_{g}^{2}$ ) $\times R=\operatorname{int} H_{2 g}^{3}$ we illustrate the case of a torus.

Figure 2(ii) shows the bounded punctured torus $T^{2}$ as two disks joined by three strips. Then $T^{2} \times I$ is obtained by thickening $T^{2}$ into two balls joined by three solid pipes. The thickness of the pipes enables us to untwist them and so establish a homeomorphism $T^{2} \times I=H_{2}^{3}$. Taking interiors we have (int $\left.T^{2}\right) \times R \cong \operatorname{int} H_{2}^{3}$. The case for higher genus is similar.

REMARK 4. The above corollary is also a digression because throughout the rest of this paper we shall confine ourselves to smooth knots. In the corollary the knots were not smooth at the suspension points. In a sense the fibering of the complement is therefore the more surprising. However, the nonsmoothness is reflected in the fact that although each fibre $F$ is an open manifold, the closure $\bar{F}$ of each fibre (obtained by adding the knot to the fibre) is not a bounded manifold. For $\bar{F}$ is the suspension of a bounded punctured torus and therefore fails to be a manifold at the two suspension points. One may describe $\bar{F}$ as the generalisation to nonsmooth knots of the concept of minimal surface spanning a smooth knot.

4. The 5-twist-spun trefoil. We shall describe intuitively how to twist-spin the trefoil into Mazur's knot. The description is repeated rigorously with formulae in $\$ 6$, and a much more general theorem is proved, but for the moment let us continue in the spirit of the last section and try to construct a picture. Firstly, the process of spinning was initiated by Artin [1] in 1925. The formula Spin $D^{1}=S^{2}$ means map the arc $D^{1}$ onto a meridian of $S^{2}$ and, keeping $\partial D^{1}$ fixed at the poles, multiply the interior of $D^{1}$ by $S^{1}$, or in other words spin the meridian about the poles to form $S^{2}$. Similarly $\operatorname{Spin} D^{n}=S^{n+1}$ means keep $\partial D^{n}$ fixed and multiply the interior of $D^{n}$ by $S^{1}$. In particular $\operatorname{Spin} D^{3}=S^{4}$.

Now in $D^{3}$ draw an arc $D^{1}$ running from the North pole to the South pole via a trefoil knot. The spinning process induces $S^{2}=\operatorname{spin} D^{1} \subset \operatorname{spin} D^{3}$ $=S^{4}$, which is the Artin knot. The additional refinement that we now add is to rotate the $\operatorname{knotted} \operatorname{arc} D^{1}$ about the polar axis relative to $D^{3}$ during the spinning. We call this process "twisting" and we actually twist 5 times during the spinning. The result is that we obtain a different knot from Artin's. For Artin's knot with zero twist has group 


$$
\begin{aligned}
\pi_{1}\left(S^{4}-S^{2}\right) & =\pi_{1}\left(D^{3}-D^{1}\right) \\
& =\text { group of the trefoil } \\
& =\{a, b ; a b a=b a b\} .
\end{aligned}
$$

Whereas the 5-twist-spun trefoil has group

$$
\pi_{1}\left(S^{4}-S^{2}\right)=\left\{a, b ; a b a=b a b, b=a^{-5} b a^{5}\right\} .
$$

The extra relation comes in as follows. Represent $b$ by a path consisting of a loop and a tail. The loop is a little loop looping the arc marked " $b$ " in Figure 3, and the tail runs from the base point to the loop. The path

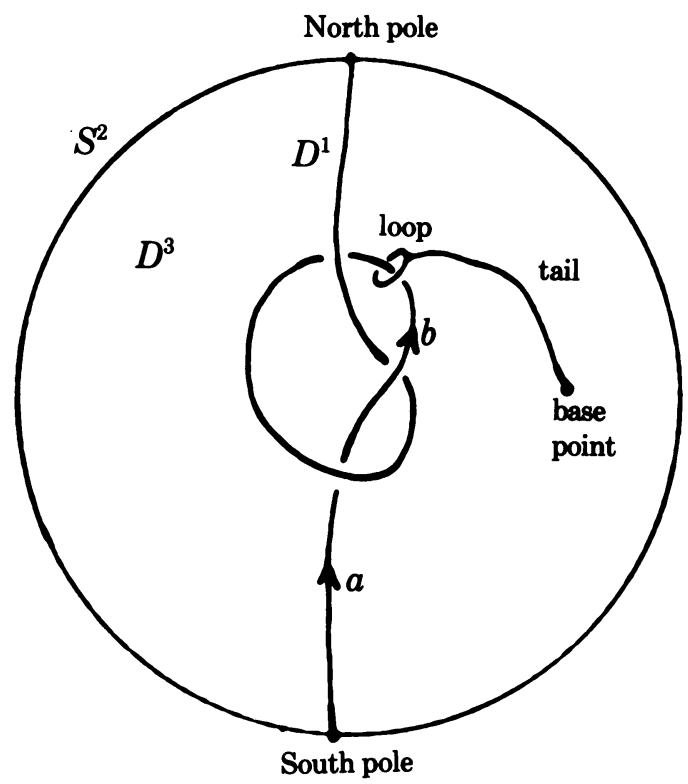

Figure 3

representing $b$ goes along the tail, round the loop, and back along the tail. Now twist-spin the loop, or more precisely homotop the loop round the twist-spin back to its original position. If we extend the homotopy of the loop to a homotopy of the path representing $b$ keeping the base point fixed of course, then the tail gets wound 5 times round the axis of twist, namely $a$. Therefore the new path represents $a^{-5} b a^{5}$, and so $b=a^{-5} b a^{5}$. Since $a^{5}$ commutes with generators $a, b$ it lies in the centre of the group. By Artin's argument [1] it can be shown that there are no other generators, and all the new relations follow from $a^{5}$ lying in the centre. We can now show that the two groups 


$$
\begin{aligned}
\pi_{1}\left(S^{4}-S^{2}\right) & =\left\{a, b ; a b a=b a b, b=a^{-5} b a^{5}\right\}, \\
G \times Z & =\left\{x, y, z ; x^{5}=(x y)^{3}=(x y x)^{2}, z^{-1} x z=y, z^{-1} y z=y x^{-1}\right\}
\end{aligned}
$$

are isomorphic by making the substitution $a=z, b=x z$. Admittedly they do not look isomorphic at first sight, and still less do they look like $G \times Z$, but this admission is an illustration of how group theory can at first sight obscure a geometrical problem rather than illuminate it. However, knowing the group itself is useful because, since it has elements of finite order, this shows that the 5-twist-spun trefoil is not the same as Artin's original 0-twistspun trefoil, because the latter has the same group as the trefoil with no elements of finite order.

We have shown that the 5-twist-spun trefoil has the same group as Mazur's knot. We shall show that they are actually the same knot, which renders our above computations redundant. However the computation has been useful in verifying the structure of the group and in relating the geometry to the structure and in detecting the elements of finite order.

We conclude this section by describing how the punctured dodecahedral spaces sit inside $S^{4}$. The fibering described in $\$ 3$ of the complement of a trefoil induces a fibering of $D^{3}-D^{1}$ by surfaces $F_{\phi}^{2}, \phi \in S^{1}$. The surface $F_{\phi}^{2}$ is in fact a "half-bounded" punctured torus, with boundary $\partial F_{\phi}^{2}=M_{\phi}^{1}$ $=$ the meridian of $\partial D^{3}$ with longitude $\phi$. The interior of $F_{\phi}^{2}$ is a punctured torus, while the closure of $F_{\phi}^{2}$ is a bounded punctured torus, whose boundary contains in addition to $M_{\phi}^{1}$ the knotted arc $D^{1}$ (see Figure 4).

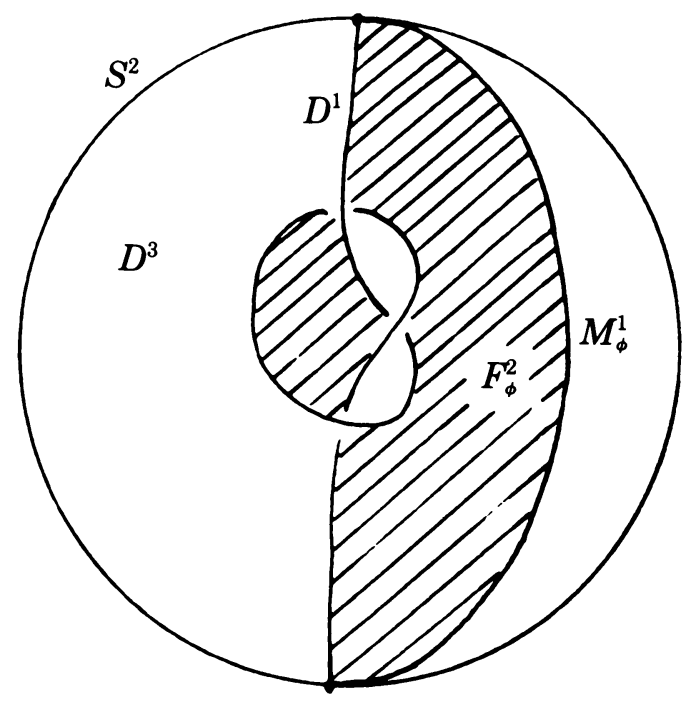

Figure 4

Given $\theta \in S^{1}$, let $D_{\theta}^{3}$ denote the position of $D^{3}$ at time $\theta$ in the spinning 
process. Let $D_{\theta}^{1}$ denote the corresponding position of the knot, which remember has been twisted through $5 \theta$ relative to $D^{3}$. Let $F_{\theta, \phi}^{2}$ denote the corresponding fibre, which since it also has been twisted through $5 \theta$ has boundary $\partial F_{\theta, \phi}^{2}=M_{\phi+5 \theta}^{1}$ (remember all the boundaries of the $D_{\theta}^{3}$ are identified). Now consider the union

$$
M_{\downarrow}^{3}=\bigcup_{\theta \in S^{1}} F_{\theta, \psi-5 \theta}^{2} .
$$

This union consists of an $S^{1}$-family of punctured tori all glued onto the single meridian $M_{\psi}^{1}$. The $S^{1}$-family of punctured tori is the 5 -fold cyclic covering of

$$
\left(\text { int } D^{3}-\operatorname{int} D^{1}\right)=\left(S^{3}-\text { trefoil }\right),
$$

and the addition of $M_{\psi}^{1}$ has the effect of adding to this covering an open arc of the branch locus in the 5-fold branched covering of $S^{3}$, branched over the trefoil. Now the 5 -fold branched covering of $S^{3}$ branched over the trefoil is none other than the dodecahedral space $M^{3}[9, \S 65]$, and puncturing the latter has the same effect as removing a tame closed arc. In particular we can remove the closed arc in the branch locus complementary to the open arc mentioned above. In other words $M_{\psi}^{3}=$ punc $M^{3}$, and as $\psi$ varies we obtain the fibering of $S^{4}-S^{2}$ over $S^{1}$. There remains to check up that this is the same as Mazur's fibering, and then we shall have established the homeomorphism between the pairs $S^{4}, S^{2}$ and Mazur's $Q^{4}, S^{2}$. We do this as follows.

We can lift the covering $R \rightarrow S^{1}$ to a covering $\nu: M^{3} \times R \rightarrow S^{4}-S^{2}$ and define the homeomorphism $h$ so that the diagram

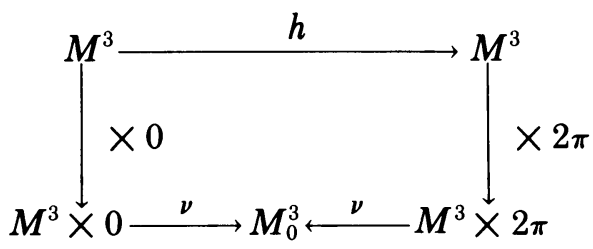

is commutative. There is a slightly subtle point here because $h$ depends upon the product structure $M^{3} \times R$ chosen in the covering space, and if we choose a different product structure (still covering the fibering below), we then obtain a different $h$, isotopic to the original one. To make sure that we get the correct $h$, of period 5 , it is necessary to resort to explicit formulae, which we do in the next section. Here it suffices to say that the product structure is equivalent to isotoping each $F_{\theta, \phi}^{2}$ onto $F_{\theta+2 \pi / 5, \phi}^{2}$ by keeping $\phi$ fixed and giving $\theta$ one fifth of a spin; this maps $M_{5 \theta+\phi}^{3}$ onto itself with the canonical covering homeomorphism of the 5 -fold branched covering, which has period 5, and can be identified with Mazur's. This establishes the homeomorphism 


$$
\left(S^{4}, S^{2}\right) \cong\left(Q^{4}, S^{2}\right)
$$

REMARK. There is another obvious looking candidate for the product structure equivalent to isotoping each $F_{\theta, \phi}^{2}$ onto itself by keeping $\theta$ fixed and giving $\phi$ a whole twist (twist as opposed to spin). At first sight this looks like the homeomorphism of the punctured torus onto itself of period 6 (rather than period 5) described in $\$ 3$; but at second sight this is not so because the homeomorphism must keep the meridian $M_{5 \theta+\phi}^{1}$ fixed, and therefore keeps the boundary of $\bar{F}_{\theta, \phi}^{2}$ fixed. But any homeomorphism of a bounded punctured torus keeping the boundary fixed cannot be periodic unless it is the identity. Hence the corresponding homeomorphism of $M^{3}$ is not periodic.

The fact that the trefoil is a Neuwirth knot, and that its complement is fibered with group $Z_{6}$ is irrelevant to the fact that the 5-twist-spun trefoil has fibered complement with group $Z_{5}$. Whatever knot we start with, the resulting $k$-twist-spun knot always has fibered complement with group $Z_{k}$. In our intuitive description above, it is true that we have made use of the fibering of the trefoil, but really this is a red herring. In Lemmas 2 and 6 it appears that the fibering is a homotopy property, and different fibrations are homotopic.

\section{Branched coverings of knots.}

Notation. Let $n \geqq 3$ and let $S^{n, n-2}=\left(S^{n}, S^{n-2}\right)$ denote a smooth knot, in other words a smooth embedding of $S^{n-2}$ in $S^{n}$. We shall always assume $n \geqq 3$ and that the embedding is smooth. Let $N$ denote a closed tubular neighbourhood of $S^{n-2}$ in $S^{n}$, and $V$ the closure of the complement,

$$
V=\operatorname{closure}\left(S^{n}-N\right) \text {. }
$$

Therefore $\partial N=\partial V$.

LEMma 1. The normal bundle of a smooth knot is trivial.

Proof. The normal bundle has group $S^{1}$ and is therefore trivial in $n \neq 4$, because then $\pi_{n-3}\left(S^{1}\right)=0$. If $n=4$, let $k \in \pi_{1}\left(S^{1}\right)$ be the characteristic class of the associated principal bundle $\partial N \rightarrow S^{n-2}$. If $k \neq 0$ the cohomology spectral sequence gives $H^{1}(\partial N)=0$. But by Alexander duality $H^{1}(\partial N)$ $\cong H_{2}\left(S^{4}-\partial N\right) \cong Z$, because $S^{4}-\partial N$ is the disjoined union of a homotopy $S^{2}$ (the interior of $N$ ) and a homology $S^{1}$ (the exterior of $N$ ). This contradiction shows $k=0$, and hence the bundle is trivial.

COROLlaRY. We can choose a homeomorphism $t: S^{n-2} \times D^{2} \rightarrow N$ such that $t(x \times 0)=x$ for all $x \in S^{n-2}$.

We use the words "tubular neighbourhood" ambiguously to denote both $N$ and $t$. 
Lemma 2. Given $S^{n, n-2}, n \geqq 4$, and given a tubular neighbourhood $t$, then there exists a map $p: V \rightarrow S^{1}$, unique up to homotopy, such that the composition

$$
S^{n-2} \times \partial D^{2} \stackrel{t}{\longrightarrow} \partial N=\partial V \stackrel{p}{\longrightarrow} S^{1}
$$

is the projection on the second factor. If $n=3$ then there exists a tubular neighbourhood $t$, for which the same property holds.

Corollari 1. $p$ can be extended to $S^{n}-S^{n-2}$ so that $p t(x \times(r, \theta))=\theta$ for all $x \in S^{n-2},(r, \theta) \in D^{2}, t>0$.

COROLlaRY 2. By approximation p can be made differentiable or piecewise linear, according to taste.

Proof of Lemma 2. Consider the first case $n \geqq 4$. There is a unique map $p_{0}: \partial V \rightarrow S^{1}$ such that $\left(^{*}\right)$ is true. Then $p_{0}$ represents a generator of $\left[\partial V, S^{1}\right]$ $=H^{1}(\partial V) \cong Z$. From the cohomology exact sequence of the pair $(V, \partial V)$, using the fact that $V$ is a homology $S^{1}$ and $n \geqq 4$, we have an isomorphism $H^{1}(V) \rightarrow \simeq H^{1}(\partial V)$. Therefore in terms of homotopy classes

$$
\left[V, S^{1}\right] \cong\left[\partial V, S^{1}\right] \text {. }
$$

Therefore $p_{0}$ can be extended to a map $p: V \rightarrow S^{1}$, which is unique up to homotopy.

Now consider the case $n=3$. The additional complication, is that $\partial V$ $\cong S^{1} \times S^{1}$ and so $\left[V, S^{1}\right] \rightarrow^{i^{*}}\left[\partial V, S^{1}\right]$ (where $i: \partial V \subset V$ ) is no longer an isomorphism, but only a monomorphism. We have to choose $t$ so that $\left[p_{0}\right]$ lies in the image of $i^{*}$ as follows. Choose $\xi \in H_{1}(\partial V)$ to generate the kernel of $i_{*}: H_{1}(\partial V) \rightarrow H_{1}(V)$. Choose $t: S^{1} \times D^{2} \rightarrow N$ such that $t\left(S^{1} \times x\right)$ lies in the class $\xi$ for some (and therefore for every) $x \in \partial D^{2}$. Define $p_{0}$ as before, and then $\left[p_{0}\right] \in\left[\partial V, S^{1}\right]=H^{1}(\partial V)$ is the Poincare dual of $\xi$.

From the commutativity of the diagram

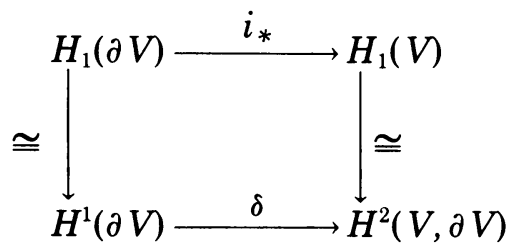

we have $\delta p_{0}=0$, because $i_{* \xi}=0$, and so $\left[p_{0}\right]$ lies in the image of $i^{*}: H^{1}(V)$ $\rightarrow H^{1}(\partial V)$ as desired. Therefore as in the previous case $p_{0}$ can be extended to $p$ and is unique up to isotopy. Lemma 2 is proved.

Definition of branched covering. Given a smooth knot $S^{n, n-2}$, we define the $k$-fold cyclic branched covering of $S^{n, n-2}$ as follows. Choose a tubular neighbourhood $t$, and a map $p: V \rightarrow S^{1}$ to satisfy $\left({ }^{*}\right)$ of Lemma 2. Let 
$k_{S}: S^{1} \rightarrow S^{1}$ be the $k$-fold covering given by $\theta \rightarrow k \theta$. Let $k_{V}: \tilde{V} \rightarrow V$ be the $k$ -

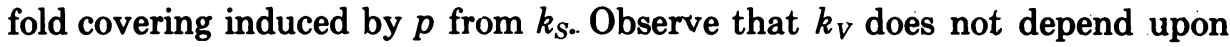
the choice of $p$, since any two choices are homotopic, and homotopic maps induce the same covering. In other words we have a commutative diagram

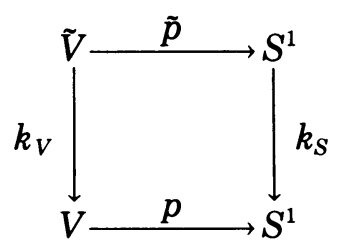

where

$$
\tilde{V}=\left\{y \times \theta ; y \in V, \theta \in S^{1}, p y=k \theta\right\} \subset V \times S^{1} .
$$

Since $\partial V=t\left(S^{n-2} \times \partial D^{2}\right)$ we have, by Lemma 2 ,

$$
\partial V=k_{V}^{-1}(\partial V)=\left\{t(x \times k \theta) \times \theta ; x \in S^{n-2}, \theta \in S^{1}\right\} .
$$

Let $e=\partial N \rightarrow \partial \tilde{V}$ be the homeomorphism given by

$$
t(x \times \theta) \rightarrow t(x \times k \theta) \times \theta, \quad x \in S^{n-2}, \theta \in \partial D^{2} .
$$

Define

$$
M^{n}=N \underset{e}{\cup} \tilde{V}
$$

We call $M^{n}$ the $k$-fold cyclic branched covering of $S^{n, n-2}$. We have already observed that $M^{n}$ does not depend upon the choice of $p$; by the theorem on the "uniqueness" of tubular neighbourhoods one can show also that $M^{n}$ does not depend on $t$.

The canonical covering homeomorphism. We shall define a homeomorphism $\kappa: M^{n} \rightarrow M^{n}$, of period $k$, whose fixed point set is the branch locus $S^{n-2}$ of the covering map $M^{n} \rightarrow S^{n}$. We call $\kappa$ the canonical covering homeomorphism. Recall that $N=t\left(S^{n-2} \times D^{2}\right)$. Define $\kappa_{N}: N \rightarrow N$ by

$$
t(x \times(r, \theta)) \rightarrow t(x \times(r, \theta-2 \pi / k)),
$$

where $x \in S^{n-2}$ and $(r, \theta)$ are polar coordinates for $D^{2}$. Define $\kappa_{V}: \tilde{V} \rightarrow \tilde{V}$ by $y \times \theta \rightarrow y \times(\theta-2 \pi / k))$. The image of $\kappa_{V}$ is contained in $\tilde{V}$ all right because $y \times \theta \in \tilde{V}$ implies $p y=k \theta=k(\theta-2 \pi / k)$. The diagram

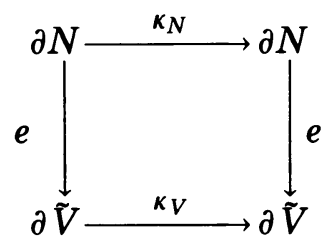


is commutative, and so the pair $\kappa_{N}, \kappa_{V}$ determine a homeomorphism $\kappa: M^{n} \rightarrow M^{n}$, which is of period $k$ since both $\kappa_{N}$ and $\kappa_{V}$ are. The fixed point set of $\kappa_{V}$ is empty, and so the fixed point set of $\kappa$ is the same as that of $\kappa_{N}$, which is $t\left(S^{n-2} \times 0\right)=S^{n-2}$.

\section{The main theorem.}

Latitude and longitude. Let $\Delta^{n}$ denote the unit ball in Euclidean $n$-space $E^{n}$. We introduce latitude and longitude coordinates in the unit sphere $\partial \Delta^{n}$ as follows. Write $E^{n}$ as the product $E^{n}=E^{n-2} \times E^{2}$. Given a point $x \in \partial \Delta^{n}$ define the latitude of $z$ to be the projection of $z$ on $E^{n-2}$, and the longitude of $z$ to be the angular polar coordinate of the projection of $z$ on $E^{2}$. Therefore the latitude of $z$ is a unique point of $\Delta^{n-2}$, and the longitude of $z$ is either a unique point of $S^{1}$ if $z \notin \partial \Delta^{n-2}$, or else is indeterminate if $z \in \partial \Delta^{n-2}$.

Definition of k-twist-spinning. Given a smooth $\operatorname{knot} S^{n, n-2}$, and an integer $k$, the $k$-twist-spinning process determines a smooth $\operatorname{knot} S^{n+1, n-1}$ one dimension higher as follows. Choose a point $x_{*} \in S^{n-2}$, and by the smoothness choose a coordinate neighbourhood $g: E^{n} \rightarrow S^{n}$ such that $g(0)=x_{*}$, and $g^{-1} S^{n-2}=E^{n-2}$, the linear subspace of $E^{n}$. Let $D_{*}^{n, n-2}=g \Delta^{n, n-2}$, the image under $g$ of the unit ball pair $\Delta^{n, n-2}$ in $E^{n, n-2}$. Let $D^{n, n-2}$ be the complementary ball pair (see Figure 1)

$$
D^{n, n-2}=\operatorname{closure}\left(S^{n, n-2}-D_{*}^{n, n-2}\right) .
$$

Then $D^{n, n-2}$ is a knotted ball pair, in effect the same knot as the given knot $S^{n, n-2}$. The boundary is unknotted because $\partial D^{n, n-2}=\partial D_{*}^{n, n-2}=g\left(\partial \Delta^{n, n-2}\right)$. Therefore we can introduce via $g$ latitude and longitude coordinates $(x, \phi)$ into $\partial D^{n}$, where $x \in D_{*}^{n-2}$ and $\phi \in S^{1}$. Let $(r, \theta)$ be polar coordinates in $D^{2}$. Let

$$
\begin{aligned}
& X=X^{n+1, n-1}=\partial D^{n, n-2} \times D^{2}=\left(\partial D^{n} \times D^{2}, \partial D^{n-2} \times D^{2}\right), \\
& Y=Y^{n+1, n-1}=D^{n, n-2} \times \partial D^{2}=\left(D^{n} \times \partial D^{2}, D^{n-2} \times \partial D^{2}\right) .
\end{aligned}
$$

Let $f: \partial X \rightarrow \partial Y$ be the homeomorphism given by

$$
(x, \phi) \times \theta \rightarrow(x, \phi+k \theta) \times \theta, \quad \text { where }(x, \phi) \in \partial D^{n}, \theta \in S^{1} .
$$

Notice that $\partial X, \partial Y$ are pairs of manifolds and that $f$ is a homeomorphism of pairs; in defining $f$ we have only mentioned coordinates in the larger manifold of each pair $\partial X^{n+1} \rightarrow \partial Y^{n+1}$ but this is all right because it induces also a homeomorphism of the smaller manifold of each pair $\partial X^{n-1} \rightarrow \partial Y^{n-1}$. This procedure of defining the map of a pair by the coordinates of the larger will be used frequently below. The purpose of $f$ is to put in the $k$ twist. Define the $k$-twist-spun knot to be

$$
S^{n+1, n-1}=X \cup Y \text {. }
$$


LeMмA 3. $S^{n+1, n-1}$ is a smooth knot, uniquely determined by $S^{n, n-2}$ and $|k|$.

Proof. We first have to verify that $X \cup_{f} Y$ is a pair of spheres. Now the homeomorphism of $\partial D^{n} \times \partial D^{2}$ onto itself given by $f$ can be extended to a homeomorphism, $f^{\prime}$ say, of $D^{n} \times \partial D^{2}$ to itself (just twist the interior of the ball $D^{n}$ along with its boundary). Hence there is a homeomorphism

$$
\partial\left(D^{n} \times D^{2}\right)=\partial D^{n} \times D^{2} \cup_{1}^{\cup} D^{n} \times \partial D^{2} \stackrel{1 \cup f^{\prime}}{\longrightarrow} \partial D^{n} \times D^{2} \bigcup_{j} D^{n} \times \partial D^{2} .
$$

Therefore the right-hand side is an $(n+1)$-sphere. Similarly the smaller one is an $(n-1)$-sphere, although a homeomorphism different from $f^{\prime}$ must be used for the smaller one in general. Therefore $S^{n+1, n-1}$ is a pair of spheres, knotted in general.

The only point where smoothness is not trivial is at the boundary between the two pairs; but by construction the smaller of each pair meets the boundary orthogonally, and so smoothness follows. To show uniqueness we must show that $S^{n+1, n-1}$ is independent of (1) choices made during the construction, and (2) the sign of $k$.

Now the only choice made in the construction is in effect the embedding $g: \Delta^{n, n-2} \rightarrow S^{n, n-2}$; any two such choices are ambient isotopic, and hence the resulting $k$-twist-spun knots are diffeotopic.

Now to deal with the sign of $k$. If $S_{+}^{n+1, n-1}$ and $S_{-}^{n+1, n-1}$ are knots corresponding to $+k$ and $-k$ respectively, then there is a homeomorphism between them given by reflecting $D^{2}$, or in other words mapping $\theta \rightarrow-\theta$ in the construction. Thus $S^{n+1, n-1}$ depends only on $|k|$, and Lemma 3 is proved.

The MAIN THEOREM. Given $S^{n, n-2}$, let $S^{n+1, n-1}$ be the $k$-twist-spun knot. Then:

1. If $k \neq 0$, there exists a bundle

$$
\left(\text { punc } M^{n}\right) \longrightarrow\left(S^{n+1}-S^{n-1}\right) \longrightarrow S^{1}
$$

with group $Z_{k}$, where $M^{n}$ is the $k$-fold cj'clic branched covering of $S^{n, n-2}$ and the generator of $Z_{k}$ is the fibre homeomorphism induced by the canonical covering homeomorphism of $M^{n}$.

2. The closure of each fibre is a smooth bounded punctured $M^{n}$ spanning the knot $S^{n-1}$ (like a minimal surface).

3. $S^{1}$ acts on $S^{n+1}$ leaving the knot $S^{n-1}$ setwise invariant, and mapping the complement fibrewise. The fixed point set is $S^{n-3} \subset S^{n-1}$, and the action of $S^{1}$ on the knot $S^{n-1}$ is to rotate it once about $S^{n-3}$.

Before proving the theorem we deduce some corollaries.

CoRollary 1. If $S^{3,1}=$ the trefoil and $k=5$ we recover Mazur's knot (see $\$ \$ 2$ and 4) and deduce that Mazur's homotopy 4-sphere $Q^{4}$ is a true 4-sphere. 
Corollary 2. If $k= \pm 1$ then $S^{n+1, n-1}$ is unknotted.

For the 1-fold branched covering of $S^{n, n-2}$ is $S^{n}$, and so $S^{n-1}$ is spanned by a smooth ball, which is a criterion for unknottedness.

Corollary 3. If $S^{n, n-2}$ is unknotted then so is $S^{n+1, n-1}$.

For the $k$-fold branched covering of an unknotted $S^{n, n-2}$ is $S^{n}$.

Consequently, we do not get any joy out of twist-spinning $S^{2,0}$, in spite of the fact that Mazur's construction can be reproduced nontrivially one dimension lower (see §3).

CoRollary 4. If $M^{n}$ is the $k$-fold cyclic branched covering of $S^{n, n-2}$ then we can embed punc $M^{n} \subset S^{n+1}$ differentially, and $M^{n} \subset S^{n+2}$ piecewise linearly (by putting a cone on the puncture hole).

Corollary 5. If $p$ odd, then the punctured lens spaces $L(p, q)$ can be embedded in $S^{4}$ differentially.

For by Schubert $[8$, Satz 6$], L(p, q)$ is the double branched covering of a knot.

7. Proof of the main theorem. We shall prove the main theorem in several steps. The first step gives the action of $S^{1}$.

Action of $S^{1}$. Recall $X=\partial D^{n, n-2} \times D^{2}, Y=D^{n, n-2} \times \partial D^{2}$. Given $\psi \in S^{1}$, define

$$
\begin{aligned}
& \psi_{X}: X \rightarrow X \quad \text { by } \quad(x, \phi) \times(r, \theta) \rightarrow(x, \phi+k \psi) \times(r, \theta-\psi), \\
& \psi_{Y}: Y \rightarrow Y \quad \text { by } \quad y \times \theta \rightarrow y \times(\theta-\psi) .
\end{aligned}
$$

The diagram

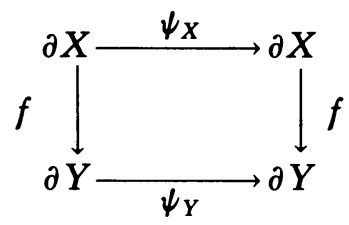

is commutative, and so the pair $\psi_{X}, \psi_{Y}$ determine a homeomorphism $\psi_{S}: S^{n+1, n-1} \rightarrow S^{n+1, n-1}$. Since $\psi_{S} \psi_{S}^{\prime}=\left(\psi+\psi^{\prime}\right)_{S}$ this is a group action. By definition $S^{n-1}$ is an invariant submanifold. If $\psi$ is not a multiple of $2 \pi / k$, then $\psi_{Y}$ has no fixed points and so the fixed point set of $\psi_{S}$ equals that of $\psi_{X}$ which is $\partial D^{n-2} \times 0$, namely the "polar" $S^{n-3}$ of $S^{n-1}$. If $\psi$ is a multiple of $2 \pi / k$ and not the identity, the fixed point set of $\psi_{Y}$ remains empty, but that of $\psi_{S}$ and $\psi_{X}$ expands to $\partial D^{n} \times 0$, which is an unknotted $(n-1)$ sphere in $S^{n+1}$ meeting the $k$-twist-spun knot $S^{n-1}$ in its polar $S^{n-3}$.

The fibering. The map $p: V \rightarrow S^{1}$ of Lemma 2 can be extended naturally inside the tubular neighbourhood to a map $p:\left(S^{n}-S^{n-2}\right) \rightarrow S^{1}$. Moreover 
we can choose the coordinate neighbourhood $g$ used in the construction of the knot to be compatible with the tubular neighbourhood $t$ used in Lemma 3 ; in other words there are embeddings $j_{1}: E^{n-2} \rightarrow S^{n-2}$ and $j_{2}: E^{2} \rightarrow D^{2}$ such that the triangle

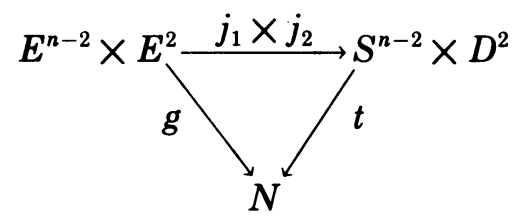

is commutative. Consequently $p$ determines a map

$$
p:\left(D^{n}-D^{n-2}\right) \rightarrow S^{1}
$$

such that $p(x, \phi)=\phi$ for $(x, \phi) \in \partial D^{n}-\partial D^{n-2}$. We use $p$ to define a projection $q$ as follows: Define

$$
\begin{aligned}
& q_{X}:\left(X^{n+1}-X^{n-1}\right) \rightarrow S^{1} \quad \text { by } \quad(x, \phi) \times(r, \theta) \rightarrow \phi, \\
& q_{Y}:\left(Y^{n+1}-Y^{n-1}\right) \rightarrow S^{1} \quad \text { by } \quad y \times \theta \rightarrow p y-k \theta .
\end{aligned}
$$

In particular if $y=(x, \phi) \in \partial\left(Y^{n}-Y^{n-2}\right)$ then $q_{Y}(y \times \theta)=\phi-k \theta$. Therefore the triangle

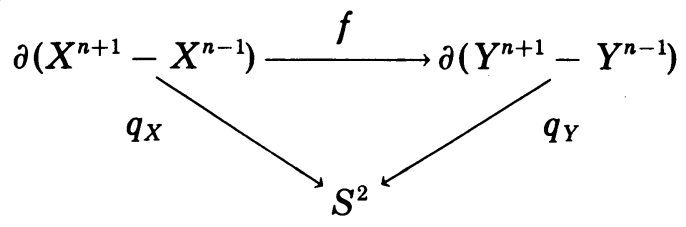

is commutative, and so the pair $q_{X}, q_{Y}$ determines a projection $q:\left(S^{n+1}-S^{n-1}\right)$ $\rightarrow S^{1}$. Since, by Lemma $2, p$ was unique up to homotopy, the same can be said of $q$.

We now show that $q$ is compatible with the action of $S^{1}$. More precisely let us introduce a new definition. Let $S^{1}$ act on $S^{1}$ with $k$-rotation; that is to say given $\psi \in S^{1}$ (the group $S^{1}$ ) let $\psi_{k}: S^{1} \rightarrow S^{1}$ be the homeomorphism (of the space $S^{1}$ ) given by $\theta \rightarrow \theta+k \psi$.

Suppose $S^{1}$ acts on a space $W$, by the homeomorphisms $\psi_{W}: W \rightarrow W$, and suppose $s: W \rightarrow S^{1}$ is a map. We say $S^{1}$ acts on $s$ with a $k$-rotation if for each $\psi \in S^{1}$, the diagram

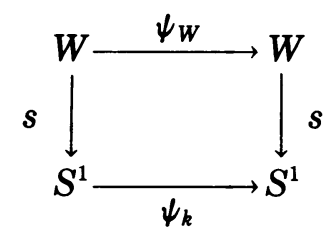

is commutative. 
Lemma 4. $S^{1}$ acts on the map $q:\left(S^{n+1}-S^{n-1}\right) \rightarrow S^{1}$ with a k-rotation.

Proof. $S^{1}$ acts on $q_{X}$ with a $k$-rotation, because, given $((x, \phi) \times(r, \theta))$ $\in X^{n+1}-X^{n-1}$, then

$$
\begin{aligned}
q_{X} \psi_{X}((x, \phi) \times(r, \theta)) & =\phi+k \psi \\
& =\psi_{k} q_{X}((x, \phi) \times(r, \theta)) .
\end{aligned}
$$

Similarly $S^{1}$ acts on $q_{Y}$ with a $k$-rotation, because given $(y, \theta) \in Y^{n+1}-Y^{n-1}$, then

$$
\begin{aligned}
q_{Y} \psi_{Y}(y \times \theta) & =p y-k \theta+k \psi \\
& =\psi_{k} q_{Y}(y \times \theta) .
\end{aligned}
$$

Therefore, combining, we have $S^{1}$ acts on $q$ with a $k$-rotation.

Lemma 5. Let $s: W \rightarrow S^{1}$ be a map on which $S^{1}$ acts with a $k$-rotation. Then $s$ is the projection of a fibre bundle with group $Z_{k}$.

Proof. Let $F=s^{-1} 0$ be the fibre above $0 \in S^{1}$. We shall choose coordinate neighbourhoods $[0, \pi]$ and $[-\pi, 0]$ in $S^{1}$, and embeddings

$$
\begin{aligned}
& c_{1}: F \times[0, \pi] \rightarrow W, \\
& c_{2}: F \times[-\pi, 0] \rightarrow W
\end{aligned}
$$

by $v \times k \psi \rightarrow \psi_{W}(v)$, where $v \in F$ and $-\pi / k \leqq \psi \leqq \pi / k$.

Then, for each $i=1,2$, we have $s c_{i}(v \times k \psi)=k \psi$, and so $c_{1}, c_{2}$ are coordinate functions for the bundle structure. To obtain the group of the bundle, it is necessary to examine the homeomorphisms of $F$ induced by the coordinate functions. There are only two homeomorphisms to consider, because the coordinate neighbourhoods overlap in only two points, namely 0 and $\pi$. The first of these is trivial, since both coordinate functions map $F$ onto the fibre $s^{-1} 0$ by the identity map. The second is nontrivial, because the coordinate functions map $F \rightarrow s^{-1} \pi$ by the homeomorphisms $(\pi / k)_{W},(-\pi / k)_{W}$, respectively.

Therefore, the induced homeomorphism $h: F \rightarrow F$ is

$$
h=\left(\frac{-\pi}{k}\right)_{W}^{-1}\left(\frac{\pi}{k}\right)_{W}=\left(\frac{2 \pi}{k}\right)_{W} .
$$

But $h^{k}=(2 \pi)_{W}=$ the identity, and so $h$ is periodic with period $k$, and the bundle group $=$ the group of homeomorphisms of $F$ generated by $h=Z_{k}$.

Corollary 1 to Lemmas 4 and 5. The map $q:\left(S^{n+1}-S^{n-1}\right) \rightarrow S^{1}$ is a fibre bundle with group $Z_{k}$. The generator $h$ of $Z_{k}$ is the homeomorphism of the fibre given by the action of $2 \pi / k \in S^{1}$.

Corollary 2. The fibres of $q$ can be made smooth by choosing $p$ to be 
differentiable (Corollary 2 to Lemma 2).

The next step in the proof is the longest: we have to identify the fibre with the punctured branched covering. In the notation of $\$ 5$ let $M^{n}$ denote the $k$-fold cyclic branched covering of $S^{n, n-2}$, and let $M_{*}^{n}=$ bounded punc $M^{n}$. Let $F$ denote the closure of the fibre $F$.

Lemma 6. There is a homeomorphism $m: M^{n} \rightarrow F$ mapping the boundary $\partial M_{*}^{n}$ onto the knot $S^{n-1}$, and the interior onto the fibre.

Proof. The notations of $\$ \$ 5$ and 6 have been chosen carefully so that they can be combined for this proof. Recall that for the definition of the branched covering $M^{n}$ we needed to choose a tubular neighbourhood $t: S^{n-2} \times D^{2} \rightarrow S^{n}$, and a function $p: V \rightarrow S^{1}$. Meanwhile for the definition of the $k$-twist-spun knot we needed to choose a coordinate neighbourhood $g: E^{n} \rightarrow S^{n}$, and for the fibering we could use the extension of $p$ to $\left(S^{n}-S^{n-2}\right)$ $\rightarrow S^{1}$ provided that $g$ was compatible with $t$; in other words the triangle

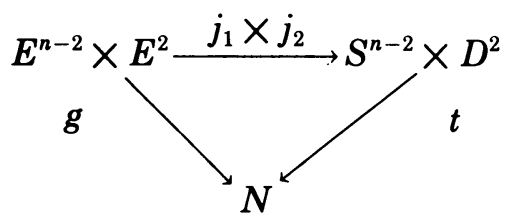

was commutative. Having arranged for the choices involved in the two constructions to be compatible, we now define the bounded punctured branched covering $M_{*}^{n}$ by removing a particular open cell from $M^{n}$, and shall then define the homeomorphism in two parts (corresponding to $X, Y$ ).

Recall the definition

$$
\begin{aligned}
& D_{*}^{n-2}=g \Delta^{n-2} \subset S^{n-2}, \\
& D^{n-2}=\operatorname{closure}\left(S^{n-2}-D_{*}^{n-2}\right) .
\end{aligned}
$$

Define $M_{*}^{n}=M^{n}-t\left(\operatorname{int} D^{n-2} \times \operatorname{int} D^{2}\right)$. We may express $M_{*}^{n}$ as the union of two parts as follows:

Let $N_{*}=t\left(D_{*}^{n-2} \times D^{2}\right)$.

Write $\partial N_{*}=\partial_{1} N_{*} \cup \partial_{2} N_{*}$ where

$$
\begin{aligned}
& \partial_{1} N_{*}=t\left(\partial D_{*}^{n-2} \times D^{2}\right), \\
& \partial_{2} N_{*}=t\left(D_{*}^{n-2} \times \partial D^{2}\right) .
\end{aligned}
$$

Write $\partial \tilde{V}=\partial_{1} \tilde{V} \cup \partial_{2} \tilde{V}$ where $\partial_{1} \tilde{V}=k_{V}^{-1}\left(\partial_{i} V\right)$ and

$$
\begin{aligned}
& \partial_{1} V=t\left(D^{n-2} \times \partial D^{2}\right), \\
& \partial_{2} V=t\left(D_{*}^{n-2} \times \partial D^{2}\right)=\partial_{2} N_{*} .
\end{aligned}
$$

Then $e$ maps $\partial_{2} N_{*}$ homeomorphically onto $\partial_{2} \tilde{V}$, and 


$$
\begin{aligned}
M_{*}^{n} & =N_{*} \cup \in V, \\
\partial M_{*}^{n} & =\partial_{1} N_{*} \cup \partial_{1} \tilde{V} .
\end{aligned}
$$

Notice that the canonical homeomorphism $\kappa$ maps $M_{*}^{n}$ to itself.

Now look at the fibre $F=q^{-1} 0$. Write $F=F_{X} \cup F_{Y}$ where $F_{X}, F_{Y}$ denote the intersections of $F$ with $X^{n+1}, Y^{n+1}$, respectively. The first part of $m$ is constructed as follows.

Let $m_{X}$ denote the embedding $m_{X}: N_{*} \rightarrow \partial D^{n-2} \times D^{2}$ given by $t(x \times(r, \theta))$ $\rightarrow(x, 0) \times(r, \theta)$, where $x \in D_{*}^{n-2}$ and $(r, \theta) \in D^{2}$.

In particular, $\partial_{1} N_{*}$ is given by restricting $x$ to values $x \in D_{*}^{n-2} \doteq \partial D^{n-2}$; and so $m_{X}$ induces homeomorphisms

$$
\begin{aligned}
& \partial_{1} N_{*} \cong \partial D^{n-2} \times D^{2}, \\
& \left(N_{*}-\partial_{1} N_{*}\right) \stackrel{\cong}{\longrightarrow} F_{X} .
\end{aligned}
$$

The second part is a little harder, and we need a sublemma.

Sublemma. There is a map $\mu: V \rightarrow D^{n}$ that maps $\partial_{1} V$ (nonhomeomorphically) onto $D^{n-2}$ and maps $V-\partial_{1} V$ homeomorphically onto $D^{n}-D^{n-2}$, such that $p_{\mu}=p$.

Proof. Let $N_{+}$be a slightly larger tubular neighbourhood than $N$, and define $\mu=1$ outside $N_{+}$. Next define $\mu: \partial_{1} V \rightarrow D^{n-2}$ by $t(z \times \phi) \rightarrow z$, where $z \in D^{n-2}, \phi \in \partial D^{2}$. Next define $\mu: \partial_{2} V \rightarrow \partial D^{n}$ by $t(\psi \times \phi) \rightarrow(x, \phi)$ where $x \in D_{*}^{n-2}, \phi \in \partial D^{2}$.

So far we have defined $\mu$ on the whole of $V$ except on the interior of the tubular annulus $N_{+}-N$; we complete the definition by extending $\mu$ radially to the tubular annulus. More precisely if $D_{+}^{2}$ denotes the standard disk of radius 2, we can extend $t: S^{n-2} \times D^{2} \rightarrow N$ to $t: S^{n-2} \times D_{+}^{2} \rightarrow N_{+}$, and so a point in $N_{+}$is of the form

$$
t(x \times(r, \phi)) \quad \text { where } x \in S^{n-2}, \phi \in S^{1} \text { and } 0 \leqq r \leqq 2 .
$$

A radius of $N_{+}$is given by fixing $x, \phi$ and varying $r, 0 \leqq r \leqq 2$. The radius meets the tubular annulus, closure $\left(N_{+}-N\right)$, in the subradius given by $1 \leqq r \leqq 2$. By extending $\mu$ radially, we may map the subradius linearly into the radius, the map being determined by the images of the end points, which are already known. If we wish, we can smooth this homeomorphism. We deduce that $\mu\left(V-\partial_{1} V\right)=D^{n}-D^{n-2}$. Outside $N_{+}$it is trivial that $p \mu=p$, because $\mu$ is the identity. Inside the tubular annulus $p$ maps $t(x \times(r, \phi)) \rightarrow \phi$, and so $p \mu=p$ because $\mu$ preserves $\phi$.

We now return to the proof of Lemma 6 . Let $m_{Y}$ denote the product map

$$
m_{Y}=\mu \times 1: V \times S^{1} \rightarrow D^{n} \times \partial D^{2} .
$$


Then $m_{Y}$ maps $\partial_{1} \tilde{V}$ homeomorphically onto $D^{n-2} \times \partial D^{2}$ because $t(z \times k \theta)$ $\times \theta \rightarrow z \times \theta$, where $z \in D^{n-2}$ and $\theta \in S^{1}$. Also $m_{Y}$ maps $\tilde{V}-\partial_{1} \tilde{V}$ homeomorphically onto $F_{Y}$, because if $(y \times \theta) \in \tilde{V}-\partial_{1} \tilde{V}$, then $y \in V-\partial_{1} V$ and so $p y=k \theta$. Therefore $\mu y \in D^{n}-D^{n-2}$ and $q(\mu y \times \theta)=p(\mu y)-k \theta=p y-k \theta$ $=0$, and so $\mu y \times \theta \in F_{Y}$.

Next we verify that the diagram

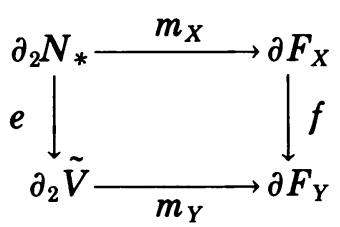

is commutative, because given $t(x \times \theta) \in \partial_{2} N_{*}$, where $x \in D_{*}^{n-2}, \theta \in \partial D^{2}$, then

$$
\begin{aligned}
f m_{X} t(x \times \phi) & =f((x, 0) \times \theta) \\
& =(x, k \theta) \times \theta \\
& =m_{Y}(t(x \times k \theta) \times \theta) \\
& =m_{Y} e(x \times \theta) .
\end{aligned}
$$

Therefore $m_{X}, m_{Y}$ combine to give a homeomorphism $m: M_{*}^{n} \rightarrow \bar{F}$ that maps

$$
\partial M_{*}^{n}=\partial_{1} N_{*} \bigcup_{e} \partial_{1} \tilde{V}
$$

onto

$$
S^{n-1}=\left(\partial D^{n-2} \times D^{2}\right) \underset{f}{\cup}\left(D^{n-2} \times D^{2}\right) .
$$

This completes the proof of Lemma 6.

Corollary to Lemma 6. If $\kappa$ is the canonical covering homeomorphism of $M^{n}$ and $h$ the homeomorphism of $F$ generating $Z_{k}$, then the diagram of homeomorphisms

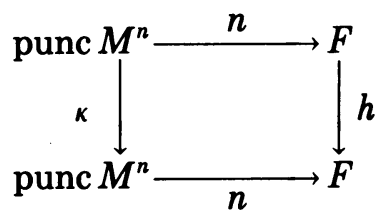

is commutative.

Proof. Given $u \in N_{*}-\partial_{1} N_{*}$, then $u=t(x \times(r, \theta))$ for some $x \in D_{*}^{n-2}$ and $(r, \theta) \in D^{2}$, and 


$$
\begin{aligned}
h m u & =h((x, 0) \times(r, \theta)) \\
& =(x, 0) \times\left(r, \theta-\frac{2 \pi}{k}\right) \\
& =m t\left(x \times\left(r, \theta-\frac{2 \pi}{k}\right)\right) \\
& =m_{\kappa} u .
\end{aligned}
$$

Given $u \in \tilde{V}-\partial_{1} \tilde{V}$, then $u=y \times \theta$ for some $y \in V-\partial_{1} V$ and $\theta \in S^{1}$, and

$$
\begin{aligned}
h m u & =h(\mu y \times \theta) \\
& =\mu y \times\left(\theta-\frac{2 \pi}{k}\right) \\
& =m\left(y \times\left(\theta-\frac{2 \pi}{k}\right)\right) \\
& =m_{\kappa} u .
\end{aligned}
$$

This completes the proof of the corollary.

Combining the corollaries to Lemmas 4, 5 and 6 completes the proof of the theorem.

\section{Questions.}

Question 1. Is there a branched covering $M^{n}$ of a knot $S^{n, n-2}$ that cannot be smoothly embedded in $S^{n-2}$ ? For if so this would give an example of a difference between differential and piecewise linear embeddings of manifolds (in view of Corollary 4 to the theorem).

Question 2. Let $S^{2} \subset S^{4}$ be Mazur's knot. Corresponding to the nontrivial bundle structure of $S^{4}-S^{2}$ there is a nontrivial group extension structure to the fundamental group $\pi_{1}\left(S^{4}-S^{2}\right)$. But the latter also has a product structure $G \times Z$. Is there a corresponding product structure $S^{4}-S^{2}$ $\cong\left(\right.$ punc $\left.M^{3}\right) \times S^{1}$ ?

Question 3. In Mazur's construction there were two choices involved, the choice of $h$ and the choice of tubular neighbourhood. Do we get $Q^{4}=S^{4}$ if we choose a different $h$ ? For example what about $h^{2}$ ? By glancing at the behaviour of $h$ and $h^{2}$ near the fixed point we see that they are not conjugate homeomorphisms. Can a new factor $i$ be introduced in the $k$-twistspinning process such that the fibre homeomorphism be not the canonical covering homeomorphism $\kappa$ but $\kappa^{i}$, where $1 \leqq i \leqq k$, analogous perhaps to torus knots?

Question 4. In Mazur's construction what happens if we choose a different tubular neighbourhood? There are three problems involved 
1. Is $Q^{4}=S^{4}$ ?

2. If so do we get the same knot?

3. How does the action of $S^{1}$ affect the knot?

The first two questions depend upon the homeotopy group of $S^{2} \times S^{1}$ (the group of homeomorphisms modulo those isotopic to the identity) because isotopic tubular neighbourhoods induce diffeomorphic surgeries. Gluck [4] has shown this group to be $Z_{2}+Z_{2}+Z_{2}$, where the first two terms correspond to orientation reversals, and the third term is represented by the homeomorphism $g: S^{2} \times S^{1} \rightarrow S^{2} \times S^{1}$ given by $(r, \phi) \times \theta \rightarrow(r, \phi+\theta) \times \theta$.

Therefore, problem 1 reduces to the single question posed by Gluck [4]: Given an $S^{2}$ knot in $S^{4}$, if we remove a tubular neighbourhood and plug it back by the homeomorphism $g$ are we left with $S^{4}$ ?

Similarly problem 2 is reduced to considering only the effect of $g$. An alternative statement of the problem is to ask if a knot is determined by its complement (Fox [3, Problem 7]). As far as I know both 1 and 2 are unsolved in general, and also unsolved in the particular case of the 5-twistspun trefoil. In the special case of the 2-twist-spun trefoil, then the answer to both 1 and 2 is yes by $[4, \S 17]$ because the knot bounds the lens space $L(3,1)$ which can be "spun" in the sense of Gluck. Similarly for the other lens spaces.

Problem 3 is answerable and illustrates a more delicate property of the product structure of the tubular neighbourhood. Recall that in $\$ 2$ we said that "provided the tubular neighbourhood is chosen correctly," then $S^{1}$ rotates the knot $S^{2}$ once about a diameter. If we now remove the tubular neighbourhood and plug it back by the homeomorphism $g^{\lambda}$, and then use the product structure to extend the action of $S^{1}$, we find that $S^{1}$ now rotates the knot $5 \lambda+1$ times. In the theorem part 3 we prove that $S^{1}$ rotates the knot once: therefore this identifies the choice of tubular neighbourhood in Mazur's construction to which our theorem refers.

\section{REFERENCES}

1. E. Artin, Zur Isotopie zweidimensionalen Flächen im $R_{4}$, Abh. Math. Sem. Univ. Hamburg 4 (1926), 174-177.

2. D. B. A. Epstein, Embedding punctured manifolds, Proc. Amer. Math. Soc. 16 (1965), $175-176$.

3. R. H. Fox, Some problems in knot theory, Topology of 3-manifolds and related topics (Proc. The Univ. of Georgia Institute, 1961), pp. 168-176, Prentice-Hall, Englewood Cliffs, N. J., 1962.

4. H. Gluck, The embeddings of two-spheres in the four-sphere, Trans. Amer. Math. Soc. 104 (1962), 308-333.

5. B. Mazur, Symmetric homology spheres, Illinois J. Math. 6 (1962), 245-250.

6. L. P. Neuwirth, The algebraic determination of the genus of knots, Amer. J. Math. 82 (1960), 791-798. 
7. V. A. Rohlin, New results in the theory of 4-dimensional manifolds, Dokl. Akad. Nauk SSSR 84 (1952), 221-224. (Russian)

8. H. Schubert, Knoten mit zwei Brücken, Math. Z. 65 (1950), 133-170.

9. H. Seifert and W. Threllfall, Lehrbuch der Topologie, Teubner, Leipzig, 1934.

10. P. A. Smith, Transformations of finite period. II, Ann. of Math. (2) 40 (1939), 690-711.

11. J. Stallings, On fibering certain 3-manifolds, Topology of 3-manifolds and related topics (Proc. The Univ. of Georgia Institute, 1961), pp. 95-100, Prentice-Hall, Englewood Cliffs, N. J., 1962.

\section{Gonville and Caius College,}

Cambridge, England 